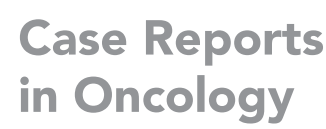

Case Reports

in Oncology

\title{
Primary Undifferentiated Pericardial Sarcoma after Radiotherapy for Hodgkin Lymphoma
}

\author{
Petr V. Glybochko Victor V. Fomin Natalia V. Chichkova \\ Evgeniya A. Kogan Andrei A. Svistunov Roman N. Komarov \\ Abram L. Syrkin \\ I.M. Sechenov First Moscow State Medical University (Sechenov University), Moscow, Russia
}

\section{Keywords}

Sarcoma of the pericardium - Undifferentiated spindle cell sarcoma - Post-radiation fibrosis of the pericardium $\cdot$ Radiotherapy $\cdot$ Hodgkin lymphoma

\begin{abstract}
Various types of sarcomas arise as a result of postradiation chronic fibrous pericarditis. A primary undifferentiated spindle cell pericardial sarcoma is a rare type of sarcoma after radiotherapy. The risk of sarcoma increases with time after treatment of cancer. A 55-year-old woman underwent successful radiation and chemotherapy for Hodgkin lymphoma 20 years ago. She was hospitalized with typical manifestations of severe heart failure. Echocardiography, computed tomography of the chest and magnetic resonance imaging scan of the heart detected neoplastic formations of the pericardium. A biopsy of the pericardium was performed. Histological, immunohistochemical, and genetic studies showed a primary undifferentiated spindle cell pericardial sarcoma (an extremely rare type of sarcoma).
\end{abstract}

(C) 2020 The Author(s).

Published by S. Karger AG, Basel

\section{Introduction}

Pericardial tumors are rare and may be classified as primary (those arising within pericardium) and secondary (those metastasizing with pericardium involvement). Primary tumors can be either benign or malignant, whereas secondary tumors are only malignant [1]. Approximately $10 \%$ tumor patients have pericardial metastases according to autopsy reports. Metastatic involvement of the pericardium is associated with leukemia, melanoma, lung, breast, and intestinal cancer $[2,3]$. Advances in such noninvasive imaging techniques as 
computed tomography (CT), magnetic resonance imaging (MRI), positron emission tomography have recently increased the frequency of primary pericardial tumor detection.

It is estimated that about $6-10 \%$ of primary heart tumors are pericardial and most of these (around 75-85\%) are benign. Among them, lipomas and various pericardial cysts occur frequently, while paragangliomas and hemangiomas are rare entities [4]. Primary malignant tumors include mesothelioma, lymphomas, various types of sarcomas (angiosarcoma, liposarcoma, synovial sarcoma, and undifferentiated sarcomas). Their prognosis is poor and associated with high mortality [1, 5-8].

Patients with some hereditary diseases (tuberous sclerosis, basal-cell nevus syndrome, neurofibromatosis type 1) and after radiation exposure have a higher risk of pericardial sarcoma. Thus, after radiation therapy, $0.017-0.17 \%$ of patients are diagnosed with sarcoma (osteosarcoma, angiosarcoma, spindle cell sarcoma) [9]. Usually, the clinical manifestations of different pericardial tumors depend on its size, location, invasion, and rate of growth but not the origin of pathological process. The most common symptoms are pericardial effusion (with or without cardiac tamponade) and congestive heart failure. More than a third of patients complain of different kinds of chest pain, shortness of breath, fainting, arrhythmias, and syncope. Sudden cardiac death may be the only manifestation of the primary pericardial tumor $[5,7]$.

\section{Case Report}

In August 2017, a 55-year-old female (patient M.) complaining of weakness, shortness of breath, and right forearm, hand and bilateral leg swelling was admitted to the therapeutic department of I.M. Sechenov First Moscow State Medical University. Her past medical history included Hodgkin lymphoma IVA (a mixed cellularity subtype, with bilateral supraclavicular, mediastinal, and left lung root node involvement; affected pericardium, left lung and pleura). A thoracotomy with the removal of the part of the left lung, bronchopulmonary lymph nodes, and the pericardial fragment was performed. Later, patient $\mathrm{M}$. was given combined chemotherapy and radiotherapy of cervical supraclavicular region, mediastinum, pericardium, and left lung. As a result, a stable remission of the disease had been achieved. Eighteen years later, in April 2015, patient M. complained of dry cough, shortness of breath, and low-grade fever. Medical examination revealed pleural effusion. Meanwhile, tuberculosis was excluded. Antibacterial therapy improved clinical sings. However, CT was not done. In autumn of 2016, a relapse of the condition with coughing and subfebrile temperature happened. A CT of the lungs showed pathological changes, regarded as post-radiation fibrosis. In spring of 2017, patient M. was hospitalized with complaints of severe dry cough and shortness of breath. CT of the lungs showed significant fibrous degeneration, pleural effusion, and infiltration of the right lower lobe. Echocardiography showed pericardial leaf seals, fibrous changes of the aortic and mitral valves. Thus, postradiation pulmonary fibrosis and cardiomyopathy, followed by lower-lobar right-sided pneumonia and heart failure were diagnosed. Treatment with antibiotics and diuretic drugs had some positive effect. However, at the end of July 2017, patient M. noted the recurrence of dyspnea, weakness, weight loss, and right forearm, hand and bilateral leg swelling. Ultrasound scan detected occlusive thrombosis of the right subclavian and jugular veins. Treatment with dabigatran was started, and the patient was admitted to our clinic.

At admission, significant swelling of the legs and feet, and swelling of the right forearm and hand attracted attention. Dullness of the percussion sound below the angle of the scapula on both sides and sharply weakened breathing during auscultation of the lungs were found. Clinical manifestations also included muffled heart sounds and tachycardia (heart rate up to 

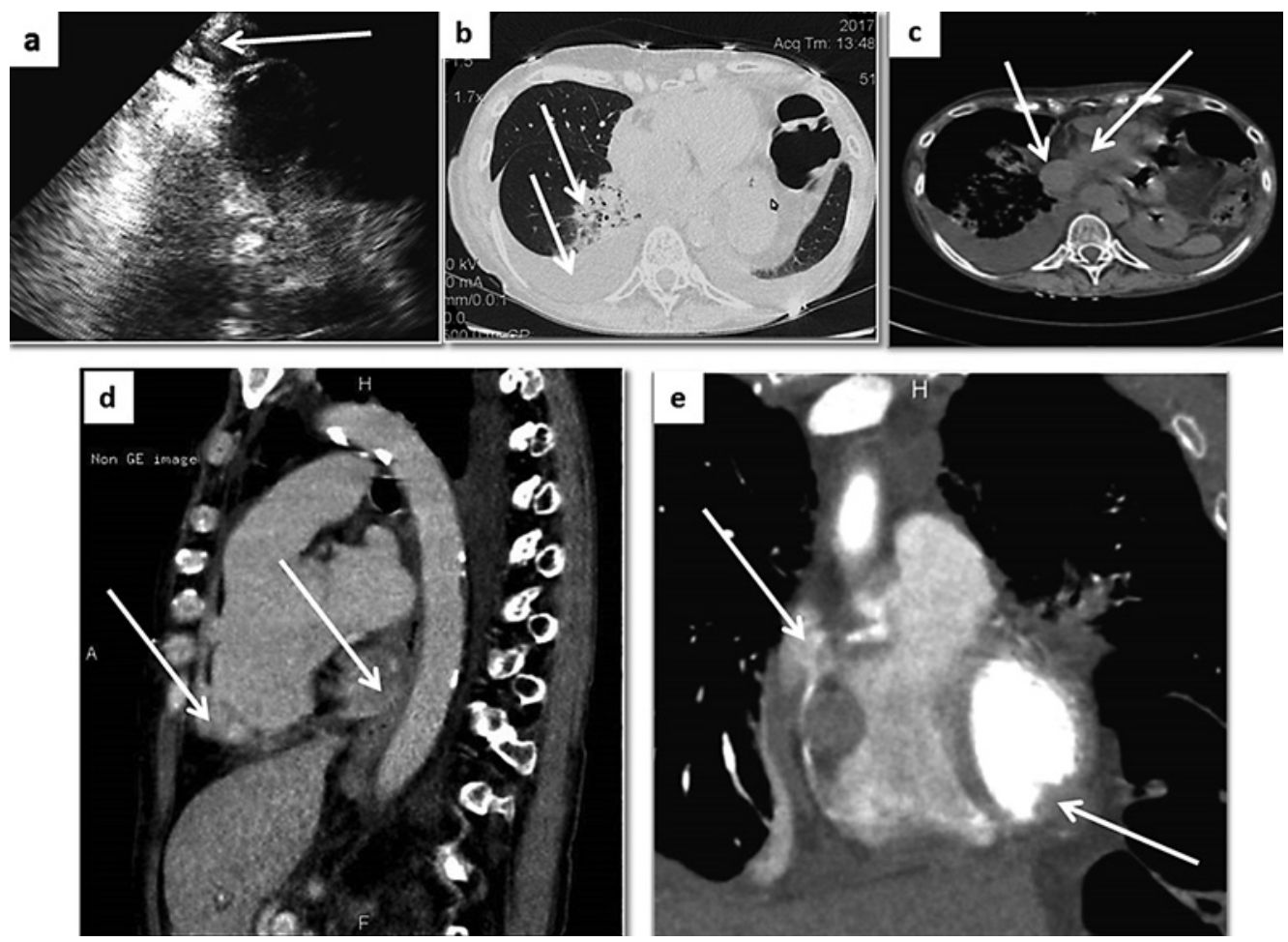

Fig. 1. Imaging findings of pericardial sarcoma and lung pathological changes. a Echocardiography. Pericardial effusion and several echo-positive formations in the left atrium, in the apical parts extending to the pericardium (arrow). b Computed tomography of the chest shows bilateral hydrothorax, multiple zones of lung fibrosis (arrows); axial image. c Contrast-enhanced computed tomography of the chest shows a node (66 $\times$ $45 \mathrm{~mm}$ ) on the back surface of the heart, which squeezes and deforms both atria and the aorta. Several nodes (up to $32 \times 23 \mathrm{~mm}$ ) are visualized on the front surface of the heart; one of them is localized in the projection of the right ventricle (arrows); axial image. d Sagittal image. e Frontal image.

$110 \mathrm{bpm}$ ). Arterial blood pressure was $120 / 80 \mathrm{~mm} \mathrm{Hg}$, the liver was enlarged by $5 \mathrm{~cm}$. Routine blood tests revealed only an increase in $\gamma$-GT to $124 \mathrm{U} / \mathrm{L}$. Electrocardiography showed a right deviation of the electrical axis of the heart, and low QRS voltage. According to echocardiography data (shown in Fig. 1 a), pericardial effusion and several echo-positive formations were determined in the left atrium. It was impossible to evaluate the function of the heart valves and myocardial contractility. The contrast-enhanced CT of the chest, abdominal cavity, and small pelvis was performed (shown in Fig. $1 \mathrm{~b}-\mathrm{e}$ ). Bilateral hydrothorax, post-radiation changes of both lungs, and thrombosis of the right subclavian and brachiocephalic veins were confirmed. Additionally, changes that could correspond to neoplastic formations of the pericardium were detected. Transudate was found in the pleural fluid.

Contrast-enhanced MRI of the heart (Fig. $2 \mathrm{a}-\mathrm{c}$ ) was performed. This study revealed a soft tissue that lined the pericardium and consisted of nodes that partially merged with each other and deformed both atria and ventricles. Since one of the nodes was fused to the aorta, the tumor growth in myocardium was possible. So, these changes were likely of pericardial lymphoma. Patient M. underwent open-chest pericardiectomy. In the anterior mediastinum, adhesions were found. Besides, in the lower third of the wound, a 7-cm formation in the ventricle was visualized. 


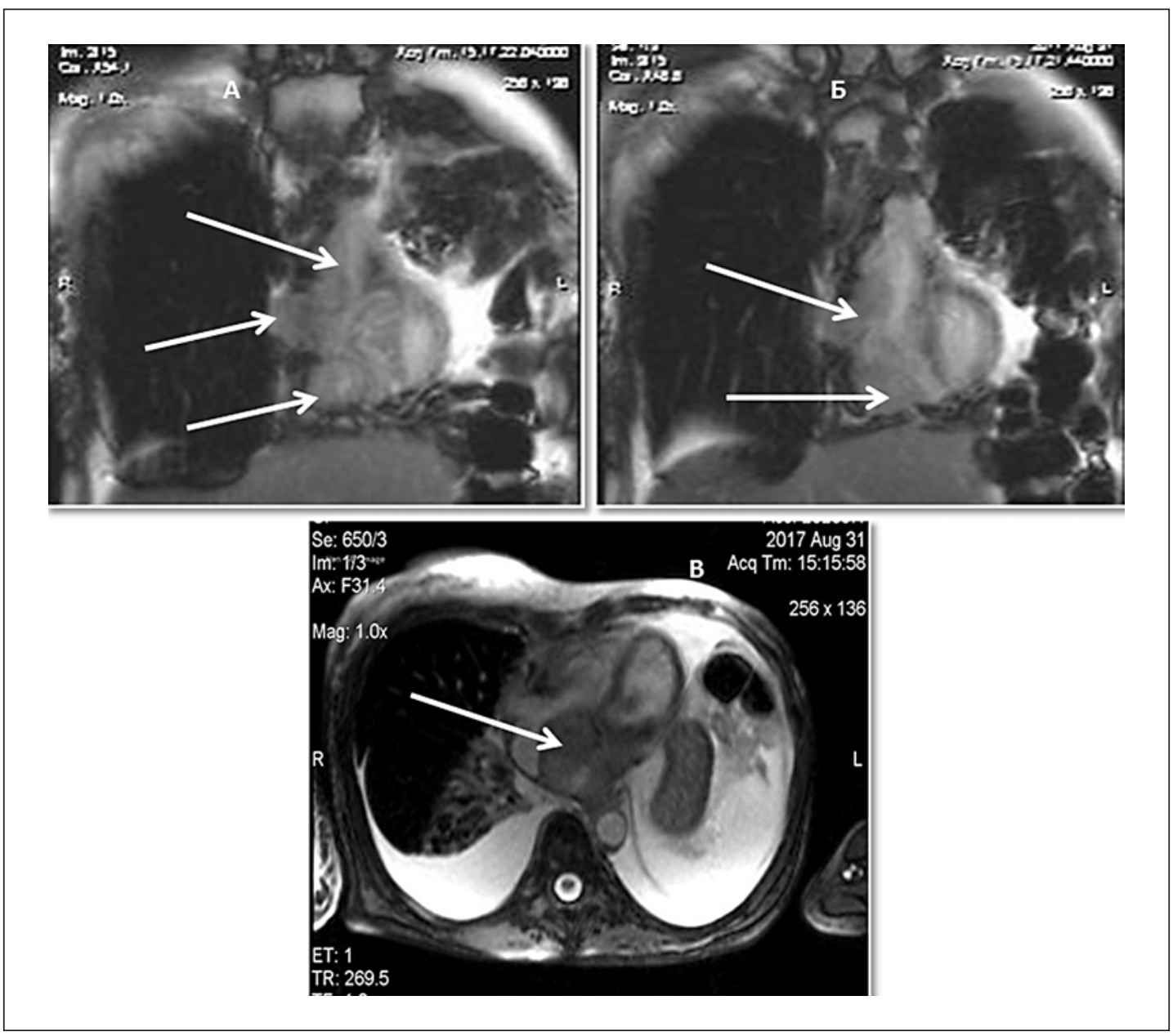

Fig. 2. Contrast-enhanced magnetic resonance imaging study of the heart. a A node on the diaphragmatic surface $(65 \times 50 \mathrm{~mm})$ which deforms left atrium and right atrium, inferior vena cava, coronary sinus, left ventricle, and right ventricle; the node is soldered to the aorta (arrows). T2-weighted imaging (T2WI) coronal image. b A node $(71 \times 55 \mathrm{~mm})$ which encases the right ventricle deforms the cavity and is not differentiated from the myocardium (arrows). T2WI coronal image. c A node, which deforms the left ventricle cavity (arrow). T2WI axial image.

Histological study showed areas of hyalinosis and fibromatous tissue. The later spread through all layers of the pericardium and located across small vessels leading to their obliteration (shown in Fig. 3 a, b). These changes were pathognomonic for radiation-induced pericardial fibrosis, which in this case was associated with radiotherapy for mediastinum in the course of Hodgkin lymphoma treatment 20 years ago.

Furthermore, in fibromatous areas, there were signs of sarcomatoid tissue growth with the spread through pericardium. Microscopically, this tumor had undifferentiated malignant spindle cells with high mitotic activity (shown in Fig. 3c). Histological signs of recurrence of Hodgkin lymphoma were not detected. To distinguish between pericardial mesothelioma, synovial sarcoma, and angiosarcoma [10], an immunohistochemical study of the tumor tissue was performed using a panel of markers: Ki67, CD30, CD45, vimentin, SMA, calretinin, CD34, and MDM2 (DAKO Cytomation). The spindle cells of the tumor did not show CD30, CD45, calretinin, or CD34 expression, but were vimentin (shown in Fig. 3d) and MDM2 (Fig. 3e) positive. So, we suspected an intimate pericardial sarcoma with increased proliferation of 


\section{Case Reports in Oncology}

\begin{tabular}{l|l}
\hline Case Rep Oncol 2020;13:1075-1081 \\
\hline DOI: 10.1159/000510068 & $\begin{array}{l}\text { @ 2020 The Author(s). Published by S. Karger AG, Basel } \\
\text { www.karger.com/cro }\end{array}$ \\
\hline
\end{tabular}

Glybochko et al.: Sarcoma of the Pericardium
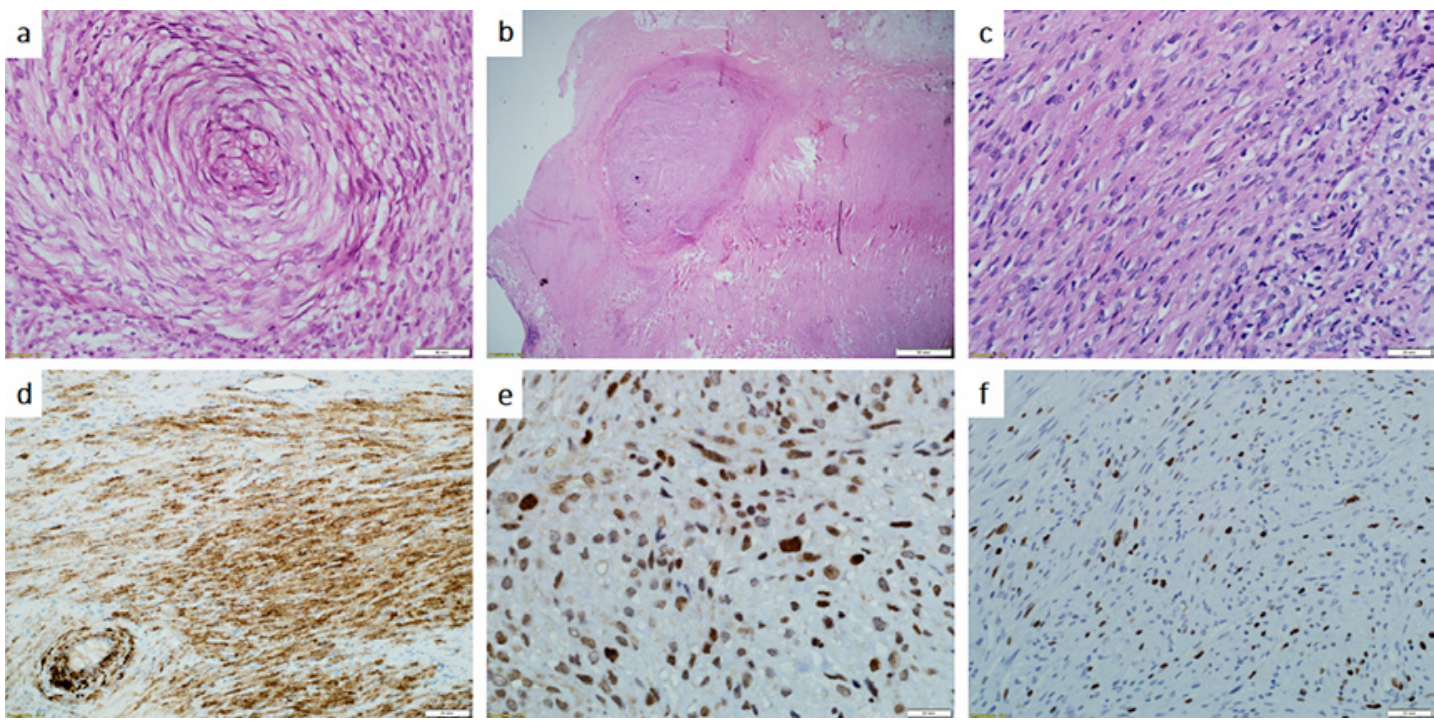

Fig. 3. Light microscopic images of pericardial spindle cell sarcoma. a Sclerosed vessel with obliteration of the lumen in the area of pericardial fibrosis; hematoxylin-eosin (HE)-stained cross section, magnification, $\times 200$. b Pericardial tissue hyalinosis fields; HE-stained cross section, magnification, $\times 100$. c Undifferentiated spindle cell pericardial sarcoma; HE-stained cross section, magnification, $\times 400$. d Immunofluorescence labeling of vimentin in cells of undifferentiated spindle-cell pericardial sarcoma; magnification, $\times 100$. e Immunofluorescence labeling of MDM2 in cells of undifferentiated spindle-cell pericardial sarcoma; magnification, $\times 600$. $\mathbf{f}$ Immunofluorescence labeling of Ki67 in cells of undifferentiated spindle cell pericardial sarcoma; magnification, $\times 400$.

Ki67 - a subtype of angiosarcoma (Fig. 3f). To narrow the differential diagnosis, fluorescence in situ hybridization on paraffin sections was performed. As a result of genetic analysis, such specific tumors as intimate sarcoma (no MDM2 amplification was detected when using MDM2 (12q15)/SE12 probe (Kreatech)), synovial sarcoma (using ON SYT (18q11) Break-Kreatech sample, no translocation of the 18q11.2 region was found), and liposarcoma (when using the LSI DDIT3 (12q13) dual color Abbott probe, no translocation of the 12q13 region was detected) were excluded. According to the histological, immunohistochemical, and genetic studies, the undifferentiated pericardial spindle cell sarcoma was diagnosed.

Because of pericardial tumor invasion in myocardium and large vessels, its surgical removal was impossible. Symptomatic therapy included the use of dabigatran, ivabradine with torasemide, and spironolactone. Patient $M$ was consulted in the chemotherapeutic department of N.N. Blokhin National Medical Research Center of Oncology. Given the severity of the condition and advancement of the tumor, it was decided not to use the standard chemotherapy. It was planned to start treatment with a tyrosine kinase inhibitor pazopanib. However, the patient's condition progressively deteriorated, and the growing cardiovascular and subsequent multiple organ failure led to the death of the patient. An autopsy was not conducted.

\section{Discussion}

Due to nonspecific diverse symptoms, primary pericardial tumors a very difficult to diagnose. The modern approach includes cardiac and blood vessel imaging [5, 7]. At the same time, this case report demonstrates that echocardiography, contrast-enhanced CT, and heart MRI show only the pericardial tumor growth. The diagnosis and treatment (the possibility of 
selecting chemotherapeutic drugs) can be made through histological, immunohistochemical, and genetic analysis.

This case report describes an extremely rare type of sarcoma - a primary undifferentiated spindle cell sarcoma of the pericardium. There are only few publications about undifferentiated cardiac sarcomas. For instance, a 32-year retrospective analysis of patients with heart tumors revealed only 4 cases of undifferentiated sarcomas with right heart localization, but without pericardial damage [6]. Agaimy et al. [11] obtained similar data in a 12-year study of the frequency of heart sarcomas in a cardiology hospital: only 3 patients with primary undifferentiated heart sarcoma were described.

The association between pericardial sarcoma and the radiotherapy for Hodgkin lymphoma is obvious. Histological study showed the growth of sarcomatoid components in fibromatous tissue. This fact confirms the impact of radiation damage, chronic inflammation, and fibrosis on the malignant tumor development [12]. However, such pathologies are very rare. Thus, Kirova et al. [13] conducted a retrospective analysis of case reports of 16,705 patients who received radiation therapy for breast cancer. Only 13 of them were diagnosed with angiosarcoma localized in the mammary gland, anterior chest wall, and skin; the average time of onset was 9.3 years from the moment of radiation exposure. There are 2 cases of pericardial angiosarcoma 8 and 15 years after radiotherapy for breast cancer [9].

\section{Conclusion}

This case report describes the development of undifferentiated spindle cell pericardial sarcoma following postradiation chronic fibrous pericarditis. This type of sarcoma may be determined only with immunohistochemical and genetic studies. Our case demonstrates extremely poor prognosis and aggressive course of the disease; thus, the long-term follow-up of patients treated with radiotherapy is needed.

\section{Acknowledgements}

We thank Dr. Karseladze A.I. (Department of Pathology, N.N. Blokhin National Medical Research Center of Oncology) for his contributions in genetic study.

\section{Statement of Ethics}

The patient's husband has given informed consent to publish this case including publication of images.

\section{Conflict of Interest Statement}

All authors declare no potential conflicts of interest related to the publication of this case report.

\section{Funding Sources}

We declare that the authors received no funds for this research.

\section{Karger'}




\section{Case Reports in Oncology}

\begin{tabular}{l|l}
\hline Case Rep Oncol 2020;13:1075-1081 \\
\hline DOI: 10.1159/000510068 & $\begin{array}{l}\text { @ 2020 The Author(s). Published by S. Karger AG, Basel } \\
\text { www.karger.com/cro }\end{array}$ \\
\hline
\end{tabular}

Glybochko et al.: Sarcoma of the Pericardium

\section{Author Contributions}

P.V. Glybochko, V.V. Fomin, N.V. Chichkova, A.A. Svistunov, R.N. Komarov, A.L. Syrkin were involved in the treatment of this patient. E.A. Kogan performed the pathological diagnosis of the tumor. N.V. Chichkova drafted the report. All authors read and critically reviewed the report. All authors approved the final submitted version.

\section{References}

1 Maleszewski JJ, Anavekar NS. Neoplastic pericardial disease. Cardiol Clin 2017;35(4):589-600.

2 Voigt P, Wienbeck S, Weber MA, Oyama-Manabe N, Beimler M, Schob S, et al. Cardiac Hematological Malignancies: Typical Growth Patterns, Imaging Features, and Clinical Outcome. Angiology. 2018;69(2): 3319717713581-75.

3 Hoffmeier A, Sindermann JR, Scheld HH, Martens S, Cardiac tumors--diagnosis and surgical treatment. Dtsch Arztebl Int. 2014; 111(12):205-11.

4 Burke A, Tavora F. The 2015 WHO classification of tumors of the heart and pericardium. J Thorac Oncol. 2015; 11(4):441-52.

5 Restrepo CS, Vargas D, Ocazionez D, Martínez-Jiménez S, Betancourt Cuellar SL, Gutierrez FR. Primary pericardial tumors. Radiographics.2013;33(6):1613-30.

6 Barreiro M, Renilla A, Jimenez JM, Martin M, Al Musa T, Garcia L, et al. Primary cardiac tumors: 32 years of experience from a Spanish tertiary surgical center. Cardiovasc Pathol.2013;22(6):424-7.

7 Siontis BL, Leja M, Chugh R Current clinical management of primary cardiac sarcoma. Expert Rev Anticancer Ther.2020;20(1):45-51.

8 Siontis BL, Zhao L, Leja M, McHugh JB, Shango MM, Baker LH, et al. Primary cardiac sarcoma: a rare, aggressive malignancy with a high propensity for brain metastases. Sarcoma. 2019;2019:1960593.

9 Sharma A, DeValeria PA, Scherber RM, Sugrue G, McCullough AE, Panse PM, et al. Angiosarcoma causing cardiac constriction late after radiation therapy for breast carcinoma. Tex Heart Inst J. 2016;43(1):81-3.

10 Rosai J, Rosai and ackerman's surgical pathology. 10th ed, USA: Elsevier Mosby; 2011. Vol. Vol. 2; p. 2271-306.

11 Agaimy A, Rösch J, Weyand M, Strecker T. Primary and metastatic cardiac sarcomas: a 12-year experience at a German heart center. Int J Clin Exp Pathol. 2012;5(9):928-38.

12 Kumar V, Abbas A, Aste J. Robbins \& cotran pathologic basis of disease 9th ed, USA: Elsevier; 2015. p. 265-340.

13 Kirova YM, Vilcoq JR, Asselain B, Sastre-Garau X, Fourquet A. Radiation-induced sarcomas after radiotherapy for breast carcinoma: a large-scale single-institution review. Cancer. 2005;104(4):856-63

\section{Karger'}

